\title{
Implantación de un sistema de gestión de la calidad: beneficios percibidos
}

\author{
Álvarez García, José* \\ Fraiz Brea, José Antonio** \\ Del Río Rama, María de la Cruz ${ }^{* * *}$
}

\section{Resumen}

En este artículo se exponen los resultados del estudio empírico llevado a cabo en 186 de las 566 empresas de alojamiento turístico en España certificada con la marca "Q de Calidad Turística". El objetivo del estudio es analizar el impacto de los beneficios percibidos por las empresas, derivados de la implantación y la posterior certificación de un Sistema de Gestión de la Calidad, considerando en el nivel de implantación de los factores críticos y los resultados obtenidos. Una vez realizada una exhaustiva revisión teórica, la metodología empleada consiste en la realización de un análisis clúster que permite contrastar y verificar las hipótesis planteadas, aplicando como paso previo el análisis factorial con la finalidad de determinar la estructura de los beneficios en este sector y obtener las puntaciones factoriales necesarias para aplicar el análisis clúster. Los resultados obtenidos ponen de manifiesto que los beneficios en este sector se agrupan en tres grupos; beneficios internos, externos de clientes y externos financieros. Por último, se ha corroborado qué cuanto mayores sean los niveles de beneficios derivados de la implantación y certificación mayores serán los niveles de implementación de los factores críticos y los resultados obtenidos por la empresa.

Palabras clave: Gestión de la calidad, Q de calidad turística, alojamiento turístico, factores críticos.

Recibido: 15-05-11. Aceptado: 29-04-13

* Profesor Asociado, Universidad de Vigo, Facultad de Ciencias Empresariales y Turismo (Campus Ourense), As Lagoas s/n 32004 Ourense, e-mail: pepealvarez@ uvigo.es

** Profesor Titular (Doctor), Universidad de Vigo, Facultad de Ciencias Empresariales y Turismo (Campus Ourense), As Lagoas s/n 32004 Ourense, e-mail: jafraiz@uvigo.es

*** Profesora Contratada Doctora, Universidad de Vigo, Facultad de Ciencias Empresariales y Turismo (Campus Ourense), As Lagoas s/n 32004 Ourense, e-mail: delrio@ uvigo.es 


\title{
Implantation of a Quality Management System: Perceived Benefits
}

\begin{abstract}
This article explains the results of an empirical study carried out in 186 of the 566 tourist lodging businesses in Spain that are certified with the seal "Q for Tourist Quality." The objective of the study is to analyze the impact of perceived benefits for businesses derived from the implantation and later certification of a quality management system, on the level of implementing critical factors and the results obtained. Once an exhaustive theoretical review had been carried out, the methodology consisted of performing a cluster analysis that made it possible to contrast and verify the proposed hypothesis. Factorial analysis was applied as a prior step to determine the benefit structure in this sector and obtain the necessary factorial scores to apply the cluster analysis. Results show that benefits in this sector fall into three groups: internal benefits, external client benefits and external financial benefits. Finally, the study corroborated that the greater the benefit levels derived from implantation and certification are, the greater the implementation levels for critical factors and results obtained by the business will be.
\end{abstract}

Keywords: Quality management, Q for Tourist Quality, tourist lodging, critical factors.

\section{Introducción}

En el sector turístico de España la estrategia de mercado seguida tradicionalmente estaba centrada en los precios, sin embargo, la actual coyuntura socioeconómica la hace inviable, produciéndose un cambio hacia estrategias enfocadas en la calidad que se basan en la diferenciación del producto ofertado. Nadie pone en duda hoy en día, que la calidad es una condición necesaria para alcanzar el éxito en los mercados turísticos, por su capacidad para mantener o incrementar por parte de la empresa su cuota de mercado y sus resultados empresariales.

Así, en la actualidad este sector se apoya en los dos pilares básicos de la competitividad empresarial, necesaria para la supervivencia de las empresas, como son la gestión de la calidad que les permite mejorar las actividades internas (calidad interna) y el rendimiento (calidad externa) (Powell, 1995; Kaynak, 2003), junto con la innovación.

Es a principios de la década de 1990, cuando el sector turístico español toma especial conciencia de la importancia de la calidad como garantía de diferenciación frente a la numerosa oferta de otros destinos turísticos emergentes competidores de España, de forma que en 1997 se crea y se otorgan las primeras certificaciones con la Marca Q de Calidad Turística y en el 2000 se crea el Instituto de Calidad Turística Española (ICTE).

El proceso de implantación de sistemas de gestión de la calidad (SGC) en las empresas del sector turístico ha sido lento, pero en la última década se ha convertido en una las prioridades dentro del turismo. Por ello, las empresas de este sector que no lo han hecho, se están planteando implementar un SGC, de forma que le permita diferenciarse y competir en un nuevo escenario caracterizado por rápidos cam- 
bios en la oferta y la demanda (Casadesús et al., 2010), al percibir la calidad como una importante herramienta, es necesario mantener o incrementar la cuota de mercado, la satisfacción de los cliente y empleados, así como, la eficiencia y la calidad de servicio al mejorar sus procesos internos (Camisón et al., 2007; Sila, 2007; Lee et al., 2009; Mak, 2011) ${ }^{1}$.

Para Hernández et al. (2004) la implantación de un sistema de gestión de la calidad es una decisión estratégica, necesaria para alcanzar el éxito en los mercados turísticos, constituye una ventaja competitiva y favorece el ingreso de las organizaciones a mercados especializados, caracterizados por ser más exigentes, pero en ellos los beneficios económicos son más altos.

Todo ello corroborado en estos últimos años por numerosas investigaciones tanto en el sector industrial (Ghobadian y Gallear, 1996; Sila, 2007; Mokhatar y Muda, 2012), como en el sector turístico (Nicolau y Sellers, 2010; Rubio-Andrada et al., 2011; Alonso-Almeida et al., 2012) que demuestran de forma empírica la relación existente entre la calidad y el éxito de las empresas, ya que la implantación de SGC permite, proporcionar a los clientes un servicio de calidad con el que satisfacerlos y con ello obtener mejores resultados financieros. Así, el éxito se traduce en la obtención de beneficios empresariales (Tarí y Molina, 2002; Heras et al.,
2004) a través de efectos sobre las operaciones, sobre los resultados económicos, sobre los trabajadores, sobre la imagen, sobre la calidad de los productos o servicios (Casadesús y Heras, 2005). En este sentido, el proceso de implantación de SGC se ve impulsado por los beneficios que de ella se derivan, puesto que la mejora continua de la calidad de los servicios prestados genera resultados positivos y medibles.

El principal motivo que ha impulsado esta investigación, es que tras una revisión de la literatura sobre el tema se ha detectado que la gestión de la calidad ha sido ampliamente estudiada en el sector industrial (Buttle, 1997; Huarng et al., 1999; Gotzamani y Tsiotras 2002, entre otros) pero existen muy pocos estudios en el sector turístico (Casadesús et al., 2010; Mak, 2011; Sheehan y Presenza, 2011; Tarí y Pereira, 2012; Tarí et al., $2012)^{2}$, sector con características únicas y muy diferentes; intangibilidad del servicio, inseparabilidad de la producción del consumo, la intensidad de la mano de obra, la heterogeneidad de los servicios, caducidad, entre otros aspectos, por lo que su calidad no puede ser gestionada de igual manera que los productos tangibles (Camisón et al., 2007).

Por todo lo expuesto, se plantea este estudio empírico por tres razones fundamentales: 1) el vacio detectado de investigaciones; 2) la importancia que el

1 Una relación más extensa de los beneficios derivados de la implantación/certificación de las normas ISO 9000 pueden consultarse en Camisón et al. (2007:772).

2 Son muy escasos y recientes los trabajos que analizan la implantación de sistemas de gestión de calidad y sus consecuencias en el funcionamiento de la empresa, en el Sector Turístico. 
turismo tiene en España (el turismo supone el $11 \%$ del PIB español en el año 2010 (INE, 2011); 3) la revisión de la literatura ha permitido detectar que los estudios en el ámbito de la gestión de la calidad se han realizado mayoritariamente en el ámbito del aseguramiento (ISO 9000) o en gestión de la calidad total (Modelo Europeo de Excelencia -EFQM), en este trabajo el estudio se centra en empresas del sector turístico que tengan implantada la Marca $Q$ de calidad turística en base a la norma UNE182001:2008-hoteles y apartamentos turísticos, marca propia del sector y única en el mundo, basada en una normativa que se sitúa en un nivel intermedio entre la ISO 9000 y el Modelo EFQM, por lo que la implantación de la misma es compatible con ambas certificaciones.

El objetivo de este trabajo es analizar el impacto de los beneficios percibidos por las empresas, derivados de la implantación y la posterior certificación de un sistema de gestión de la calidad, en el nivel de implantación de los factores críticos y los resultados obtenidos. Para ello, se identifican los beneficios que perciben los establecimientos hoteleros de la implantación de la " $Q$ de Calidad Turística"; se analizan si las características de los establecimientos hoteleros, como son el tamaño, la categoría del establecimiento, subsector de pertenencia, las certificaciones que poseen, y según la antigüedad en la certificación " $Q$ de Calidad Turística", influyen en los beneficios percibidos; $y$, se determina la estructura de los beneficios en el sector de alojamiento turístico.

El ámbito de estudio son las empresas del sector de alojamiento turístico que posean la certificación " $Q$ de Calidad Turística" basada en la norma UNE UNE182001:2008-hoteles y apartamentos turísticos, por su importancia dentro del sector turístico ${ }^{3}$ y por ser el subsector con mayor número de certificaciones.

Para elaborar la base de datos se empleó la información obtenida de la página web del Instituto para la Calidad Turística Española -ICTE- (www.icte.es). La población objetivo quedó constituida por 566 empresas de alojamiento que poseen la certificación " $Q$ de Calidad Turística" a nivel nacional, adheridas al ICTE en el año 2010. Se envió a través de correo electrónico los cuestionarios y fueron devueltos debidamente cumplimentados 164, mientras que 22 estuvieron incompletos. En este caso se solicitó a través de correo electrónico y contacto telefónico la cumplimentación de los mismos, lo que proporcionó una muestra de 186 cuestionarios válidos que representan un

3 Según la Organización Mundial del Turismo (OMT), la contribución del turismo a la actividad económica mundial en el año 2011 se estima en cerca del 5\% (10\% del PIB en España en el 2011), su contribución al empleo se estima entre el $6 \%$ y el $7 \%$ del número total de empleos en todo el mundo (directos e indirectos), 2.7 millones de empleos en España; casi el 50\% se concentra en hostelería. El sector de hostelería, según datos del 2010 , representa algo más del 0,8 por ciento de la producción total española y es en la actualidad un sector de importante valor económico en la estructura económica española, que alcanza valores próximos en torno al $7,2 \%$, en consideración con el valor añadido bruto. 
índice de respuesta de un $32,86 \%$ y un error muestral del $6,01 \%$ a un nivel de confianza del $95 \%(Z=1,96, p=q=0,5)$.

En cuanto al tamaño o dimensión de las entidades la mayor parte de las mismas corresponden a pequeñas empresas $53,8 \%$ (0-49 trabajadores) y las medianas representan el 46,2\% (50 a 249 trabajadores), 100 y 86 empresas respectivamente. Si se mide el tamaño por el número de habitaciones el $59,1 \%$ de los establecimientos tienen 100 o menos habitaciones, el $35,5 \%$ tienen más de 100 y menos o igual a 300 y el $5,4 \%$ tienen más de $300(110,66$ y 10 empresas respectivamente). Si se hace referencia a la categoría del establecimiento hotelero 13 (7\%) poseen 1-2 estrellas, 64 (34,4\%) son de 3 estrellas y $109(58,6 \%)$ tiene la categoría de 4-5 estrellas. Existen 65 $(34,9 \%)$ empresas certificadas con una antigüedad menor o igual a 3 años en la norma UNE 182001:2008 (hoteles y apartamentos turísticos), 59 (31,72\%) empresas con una antigüedad mayor de 5 y menor o igual a 5 años $127(68,27 \%)$ empresas.

Paralelamente a la selección de la población objetivo, se diseñó un cuestionario el cual permitirá conocer los beneficios percibidos por las empresas de alojamiento turístico derivados de la implantación y certificación de la " $Q$ de Calidad Turística". Se realizó una revisión bibliográfica de los trabajos de investigación en este ámbito de estudio (Adanur y Allen,
1995; Jones et al., 1997; Buttle, 1997; Tarí y Molina, 2002; Casadesús y Karapetrovic, 2005; Casadesús y Heras, 2005, entre otros), centrada esta en los ítems empleados para medir los beneficios percibidos, con el propósito de que el cuestionario cumpliera en lo posible el requisito de validez interna, de esta forma el uso repetido de los ítems para medir los beneficios percibidos por las empresas nos garantiza dicha validez ${ }^{4}$.

El resultado de este proceso fue la obtención de un cuestionario en el que los beneficios serán medidos a través de 15 ítems, utilizando para ello una escala Likert de 7 puntos para la medición de las variables en términos cuantitativos (1nada importante a 7- muy importante). La medición se realizó a través de las percepciones de los directivos que cubren la encuesta (186 empresas), a los que se les preguntó acerca del impacto con que habían percibido dichos beneficios.

\section{La $Q$ de calidad turística: fundamentos teóricos}

La marca $Q$ de calidad turística se crea en 1997 en España, constituyéndose como un sistema de gestión de la calidad propio y único en el mundo para el sector turístico, compuesto por cuatro componentes (Casadesús et al., 2010: 607): 1) normas de calidad especificas para cada unos de los subsectores turísticos, que definen el proceso, los estánda-

4 La validez interna supone que el cuestionario debe ser un instrumento de medida lo más fiel posible, que no deforme la realidad (Lambin, 1990:166) e indica el grado en el cual el proceso de medición está libre tanto del error sistemático como del error aleatorio (Kinnear y Taylor, 1995). 
res de servicio y los requisitos de calidad del mismo; 2) un sistema de certificación mediante el cual una tercera parte independiente garantiza que las empresas cumplan las normas; 3 ) la Marca $Q$ de Calidad Turística; 4) un organismo de gestión, conocido como el Instituto de Calidad Turística Española (ICTE) ${ }^{5}$ que promueve el sistema y es responsable de su ejecución, de la integridad y difusión.

La Marca Q de Calidad Turística es otorgada por el ICTE y hasta este momento se han desarrollado las normas de calidad en 21 subsectores turísticos. La norma aplicable a los alojamientos es UNE182001:2008-hoteles y apartamentos turísticos, SGC intermedio entre el aseguramiento (ISO 9001) y calidad total (EFQM), la implantación de la misma es compatible con ambas certificaciones, estando la diferencia entre ambas certificaciones en los requisitos aplicables y el nivel de exigencia de las normas de referencia. Esta norma estandariza, con el objetivo de la búsqueda de la satisfacción del cliente, desde el sistema de gestión, hasta la prestación del servicio, pasando por la infraestructura y equipamiento.

Aunque la " $Q$ de Calidad Turística" e ISO 9000, son compatibles entre sí, no se trata de dos sistemas idénticos ${ }^{6}$. Camisón et al. (2007:618) identifican una serie de diferencias entre ambos: (1) las normas del ICTE consideran los requisitos y recomendaciones de la norma ISO 9001; (2) la norma ISO 9001 es específica para la implantación de un Sistema de Gestión de la Calidad y son sistemas puros de aseguramiento de la calidad (no marcan criterios de funcionamiento ni niveles de servicio), mientras que las normas del ICTE incluyen las especificaciones de calidad del servicio que debe implementar la empresa que se adhiera al sistema; (3) la norma ISO 9001 es más versátil y aplicable a cualquier organización mientras que las normas del ICTE sólo son de aplicación a la actividad turística; (4) desde el punto de vista del cliente, la certificación ISO 9001 no garantiza un nivel de calidad concreto sino que el servicio se ajustará a las especificaciones marcadas por el establecimiento, mientras que las normas ICTE informan del nivel de calidad esperable (Camisón et al., 2007).

En este sentido a mediados de los años '90 aparecen las primeras investigaciones sobre los beneficios ${ }^{7}$ aportados por las certificaciones, siendo los resultados de las mismas confusos y dispares (Casadesús et al., 2001). Al respecto, Ca-

5 Organismo español, privado, independiente y sin ánimo de lucro. Tiene como funciones básicas la normalización, implantación, certificación y promoción de la Marca Q de Calidad Turística.

6 La reducida tasa de penetración de las normas ISO 9000, explicada ésta por los elevados costos de la misma y la carencia de personal especializado en las Pymes turísticas (Camisón y Yepes, 1994), ha propiciado que la implantación del aseguramiento de la calidad se lleve a cabo a través de estándares propios sectoriales (Camisón et al., 2007).

7 En la revisión sobre el ámbito de estudio hemos constatado que los motivos y los beneficios de implantar un Sistema de Gestión de la Calidad son los mismos, sin embargo, es diferente el contexto, los motivos son previos a la implantación y los beneficios se generan una vez implantado. 
sadesús et al. (2004:64) afirman que "ello no se debe a su carencia de importancia, sino a la dificultad de valoración de estos, en especial en la utilización de unas variables objetivas que representen los verdaderos efectos de la certificación ISO 9000".

Dicha dificultad viene agravada por el hecho de que la calidad no ejerce un efecto directo sobre el resultado global de la organización, puesto que los factores intermedios a través de los que opera la calidad como son la productividad, la satisfacción del cliente o la imagen de la empresa, se ven influenciados por muchos otros factores que dificultan poder establecer una relación transparente entre la calidad y el resultado financiero (Hardie, 1998).

Una gran parte de los estudios se han centrado en analizar los efectos de la certificación en los resultados económicos-financieros (Hendrichs y Singhal, 2001a y b; Sharma, 2005) y sobre la rentabilidad de la empresa destacan los estudios de Häversjö (2000); Benner y Veloso (2008); Dick et al. (2008). Estos estudios utilizan datos objetivos, valoran los beneficios a partir de las diferencias de valor de las empresas antes o después de la certificación (Aarts y Vos, 2001) o bien utilizan para dicho análisis los datos contenidos en bases de datos comerciales con el objetivo de analizar la incidencia de la implantación y certificación tanto en la rentabilidad como en las ventas de las empresas (Häversjö, 2000; Wayhan et al., 2002).

Otros autores se han centrado en analizar los efectos sobre la satisfacción de los clientes (Rahman, 2001), sobre la productividad (Teerlak y King, 2006), aumento de la cuota de mercado entre otros, basándose en datos de carácter subjetivo. Para llevar a cabo sus investigaciones obtienen los datos a partir de encuestas o entrevistas cara a cara con los responsables de calidad de las empresas, evaluándose únicamente el comportamiento y percepción de la empresa en un determinado momento de tiempo (Brown y Van der Wiele, 1995).

Uno de los primeros intentos de realizar una clasificación de los beneficios fue el llevado a cabo por Buttle (1997), que los agrupa en cuatro grandes grupos: beneficios en rentabilidad, en mejora de procesos, en marketing, entre otros. En su estudio identifica que la certificación ha supuesto una mejora de los procesos así como una mejora de la imagen de la empresa y sus acciones de marketing.

Otros investigadores como Leung et al. (1999) identifican aspectos externos parecidos a los de Buttle (1997) pero, los identifican no con el mercado sino con un aumento de las demandas de los consumidores; y Jones et al. (1997) dividen las motivaciones y beneficios de la implantación del modelo ISO 9000 en dos grupos de razones, las que implican un desarrollo de la organización, como son la mejora de los procesos o la competitividad y las que no implican un desarrollo como son los requerimientos de los clientes, las acciones de marketing y las relaciones públicas.

Siguiendo a Martínez (2006) se pueden clasificar en tres grupos; beneficios organizativos y de control, beneficios en la productividad y en los costos y beneficios comerciales (Cuadro 1) y a Marín et al. (2007), Marín (2009) y Marín y Gimeno (2010) en resultados de calidad, resultados operativos y resultados económico- financieros. 


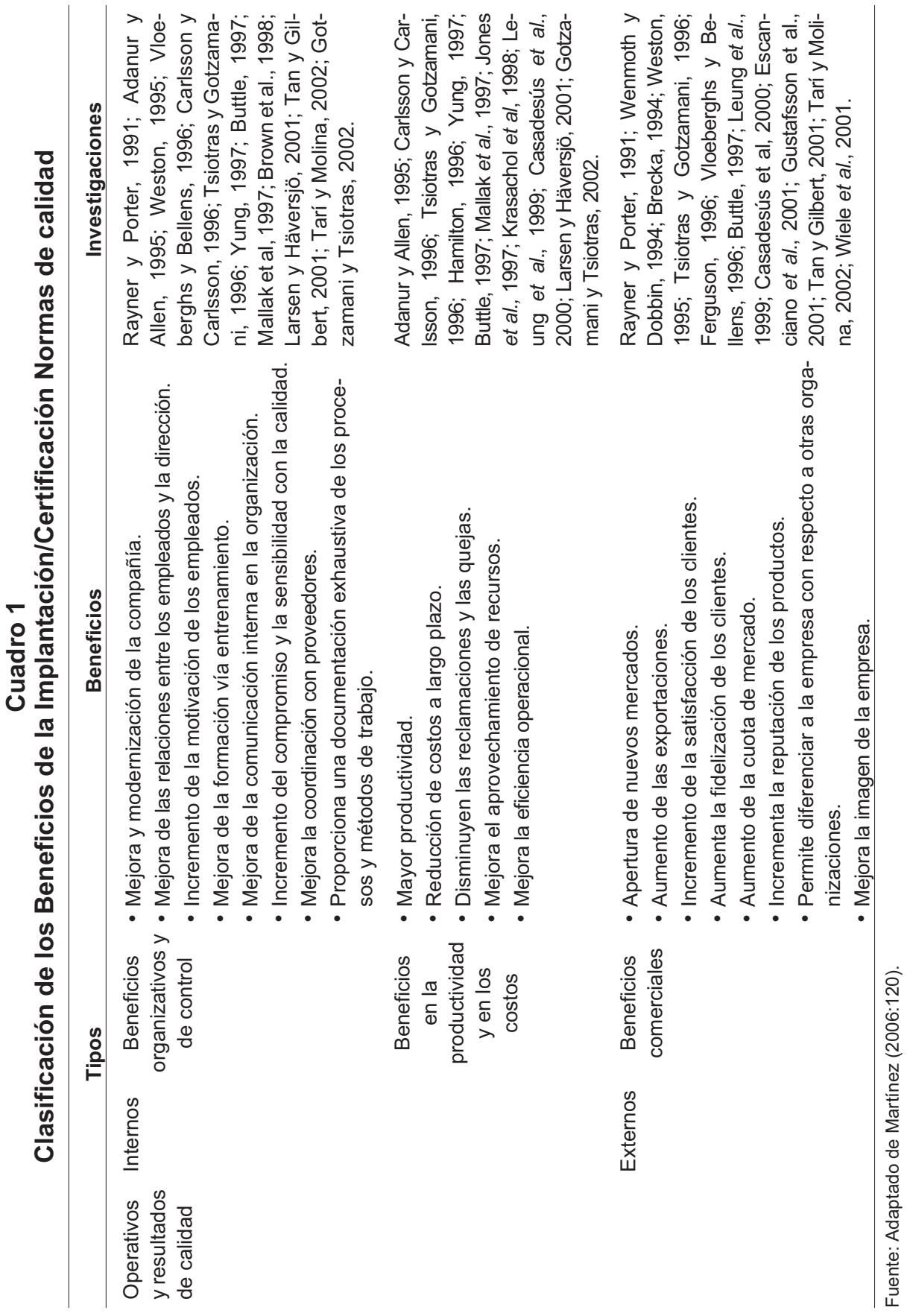


Estas investigaciones, así como los intentos de agrupar los beneficios han sido llevados a cabo utilizando dos marcos de referencia diferentes. Por un lado, el Sistema de Gestión de la Calidad ISO 9001 (Buttle, 1997; Vloeberghs y Bellens, 1996; Carlsson y Carlsson, 1996; Mallak et al., 1997; Brown et al., 1998; Larsen y Häversjö, 2001; Tan y Gilbert, 2001; Tarí y Molina, 2002; Gotzamani y Tsiotras, 2002), pero otros autores han utilizado como marco el Modelo de autoevaluación EFQM, como Camisón, 1996; Hendricks y Singhal, 1996; Eskildsen et al., 2001; Wongrassamee et al., 2003; Heras et al., 2006; Camisón et al., 2007, entre otros.

Se destaca, el estudio llevado a cabo por Heras et al. (2006) quienes "identifican cinco razones para implantar el modelo EFQM que se convierten en beneficios organizativos: decisiones corporativas, mejora de la motivación de las personas de la organización, creación de un marco estratégico, mejora de la organización y la gestión, y mejorar la imagen de la empresa. Estos autores indican que la implantación del modelo EFQM se debe principalmente a razones internas, puesto que no es una certificación reconocida a nivel internacional" (Santomá, 2008:80).

Por su parte, Camisón et al. (2007:731) en la misma línea afirman que existen muchos estudios ${ }^{8}$ que han de- mostrado que las empresas que implanten un modelo EFQM mejorarán sus resultados y además conseguirán los siguientes beneficios: 1) determinar cuáles son las fortalezas y debilidades de la organización así como una medida de la evolución del progreso de la compañía, 2) mejor determinación de las personas de la organización al entender cuál es su papel en la mejora continua de la organización, 3) un mayor compromiso de las personas de la organización al entender cuál es su papel en la mejora de la organización, 4) determinar un lenguaje común y unos criterios únicos a la hora de determinar la mejora de la organización, 5) integrar la mejora continua en la gestión operativa de la compañía, 6) aprendizaje organizativo, y 7) en caso de obtención del premio, la organización consigue un reconocimiento.

En el caso particular de los beneficios del Modelo EFQM, la mayoría de estudios indican como un beneficio fundamental que el modelo permite distinguir claramente las fortalezas y debilidades de la organización, poniendo especial enfoque en las relaciones existentes entre el personal, los procesos y los resultados (Wongrassamee et al., 2003). Estos autores indican que el modelo EFQM es una guía utilizada por la dirección de la empresa para conseguir la mejora continua y que el modelo permite a las organizacio-

8 Hendrichs y Singhal (1997) llevaron a cabo un estudio en 600 compañías que habían obtenido algún premio a la calidad y comparan sus resultados quinquenales con los de otra serie de empresas del mismo sector y tamaño. Sus resultados mostraban que las empresas que obtenían un premio a la calidad tenían mejores resultados financieros y por tanto la implantación de modelos de calidad resultaba rentable. 
nes autoevaluarse y así comprender su posición actual y planificar la mejora continua.

De los estudios se deriva que la calidad puede influir en el rendimiento de dos formas complementarias (Reed et al., 1996) por un lado a través de los procesos (efectos internos) lo que contribuirá a aumentar los niveles de competitividad y su rentabilidad al normalizar los procesos, reducción de residuos, servicio más eficaz y menos errores. Por otro, a través del mercado (efectos externos) (Brown et al., 1998; Singels et al., 2001; Yahya y Goh, 2001), ya que la calidad afectará a la satisfacción del cliente y por lo tanto su demanda lo que permitirá incrementar las ventas y cuota de mercado, $y$ tener una mejor imagen.

La mayor parte de los estudios sobre los beneficios derivados de la implantación de un SGC se han realizado mayoritariamente en el sector industrial, por lo que se considera necesario su estudio en el sector turístico y más concretamente en el sector de alojamiento. Por orto lado, existen muy pocos estudios cuyo ámbito de estudio sea la $Q$ de calidad turística. Se puede citar el llevado a cabo por Álvarez et al. (2011), en el que analizaron las barreras a las que se enfrentan los balnearios españoles en su proceso de implantación de la Q; Tarí y Pereira-Moliner (2012) que analizaron la influencia de la calidad en la rentabilidad de las cadenas hoteleras y el de Viada-Stenget et al. (2010) que analizó la implementación de un sistema de gestión de la calidad (estándar Q de Calidad Turística) en el caso del sector hotelero.

\section{Beneficios derivados de la implantación de un SGC en empresas del sector turístico español}

Antes de proceder a dar respuesta al objetivo de esta investigación; analizar el impacto de los beneficios percibidos por las empresas estudiadas, derivados de la implantación y posterior certificación de un SGC, se realiza un análisis previo en profundidad de los beneficios.

A partir de los resultados del cuestionario aplicado se realizó un análisis descriptivo que permite señalar que las mejoras más importantes percibidas por las empresas son según los resultados indicados en la Tabla 1: una clara definición de procesos y responsabilidades dentro de la organización $(6,2581)$, la mejora de la satisfacción del cliente $(6,1290)$ junto con el incremento de la conciencia de calidad de los empleados $(6,1290)$ y el mejor conocimiento de las expectativas del cliente con una media de 6,0215.

Como afirma Casadesús et al. (2007:320) "la satisfacción del cliente podría acrecentarse al conseguir la empresa alcanzar y mantener la calidad deseada, asegurando así la prestación de servicios orientados a la satisfacción de las expectativas de los consumidores. La certificación se defiende así como un instrumento adecuado para dar respuesta a los requerimientos de los consumidores, satisfaciendo sus expectativas (Mallak et al., 1997) e incluso anticipando sus necesidades futuras (Wenmoth y Dobbin, 1994)".

En este sentido, Brown y Van der Wiele (1995) y Vloeberghs y Bellens (1996) afirman que la mejora de la efi- 
Tabla 1

Beneficios obtenidos de la implantación y certificación de la $Q$ de Calidad Turística

\begin{tabular}{|c|c|c|c|}
\hline Beneficios $\left(\propto\right.$ de Cronbach $\left.^{9}=0,928\right)$ & Media & $\begin{array}{l}\text { Desviación } \\
\text { típica }\end{array}$ & $\begin{array}{c}\text { Muy } \\
\text { importante } \\
\text { (puntuación } \\
\text { entre } 5 \text { y } 7 \text { ) } \\
\% \text { empresas }\end{array}$ \\
\hline (BF2) Clara definición de procesos/responsabilidades & 6,2581 & 0,86247 & 96,8 \\
\hline (BF1) Mejora la satisfacción del cliente & 6,1290 & 1,02114 & 91,4 \\
\hline $\begin{array}{l}\text { (BF8) Incremento de la conciencia de calidad de los } \\
\text { empleados }\end{array}$ & 6,1290 & 0,80170 & 97,3 \\
\hline (BF5) Mejor conocimiento de las expectativas del cliente & 6,0215 & 1,00247 & 91,9 \\
\hline (BF12) Servicios de calidad & 5,9731 & 0,90901 & 94,1 \\
\hline (BF6) Mayor participación de los trabajadores en gestión & 5,9140 & 0,89026 & 93,5 \\
\hline $\begin{array}{l}\text { (BF9) Productividad/ mejor aprovechamiento de tiempo } \\
\text { y recursos }\end{array}$ & 5,9032 & 0,91904 & 92,5 \\
\hline (BF7) Mejora las relaciones dirección empleado & 5,7473 & 1,09821 & 87,6 \\
\hline (BF15) Posición competitiva & 5,6129 & 1,04527 & 86,6 \\
\hline (BF3) Mejora en el ambiente de trabajo & 5,5806 & 1,05853 & 84,4 \\
\hline $\begin{array}{l}\text { (BF4) Incremento de la motivación/ satisfacción de los } \\
\text { empleados }\end{array}$ & 5,5484 & 1,00287 & 86,6 \\
\hline (BF10) Reducción de quejas & 5,5323 & 1,03010 & 80,6 \\
\hline (BF14) Cuota de mercado & 5,2903 & 1,12062 & 73,1 \\
\hline (BF13) Ventas & 5,2796 & 1,15188 & 71,0 \\
\hline (BF11) Reducción de Costos & 4,9355 & 1,25035 & 64,5 \\
\hline
\end{tabular}

Fuente: Elaboración propia.

9 Este coeficiente evalúa la consistencia interna de la escala a través de la correlación de cada una de las variables con el resto de la escala. De forma generalizada la literatura utiliza este estadístico como medida de la fiabilidad (Nunnally, 1998), recomendando un valor estadístico superior a 0,8 (Grande y Abascal, 1999). La fiabilidad hace referencia al grado en que una medida se encuentra libre de errores aleatorios y, por tanto, proporciona resultados consistentes si se realizan mediciones repetitivas (Sánchez y Sarabia, 1999:367), es decir, evalúa si genera los mismos resultados en sucesivas aplicaciones a los mismos individuos, así como en situaciones similares (Babbie, 1995). 
ciencia del sistema de calidad es el beneficio más importante de implementar la ISO. Jones y Arndt (1997) afirmaron que es de esperar que tras la implantación y certificación una empresa experimente como mínimo una mayor coherencia en sus prácticas y procedimientos operacionales, y satisfacción del cliente. Escanciano et al. (2001: 198), en su estudio afirma que "las empresas que apuestan por la calidad total obtienen mayores beneficios, tanto en cantidad como intensidad, que aquellas que se limitan a conservarla o no se plantean acciones de cara al futuro en relación a la calidad".

Los beneficios menos valorados han sido la reducción de costos y el incremento de las ventas y cuota de mercado, es decir, las empresas han percibido en menor medida una mejora de estos aspectos. Todas ellas están muy bien valoradas, no presentando ninguna variable media por debajo del 4 , consideradas mejoras poco importantes.
Al comparar estos resultados con estudios previos como los realizados por Ragothaman y Korte (1999), Van der Wiele et al. (2000), Dick et al. (2001), Dissanayaka et al. (2001), Stevenson y Barnes (2001), Yahya y Goh (2001) y McAdam y Fulton (2002), Magd y Curry, 2003, que estudian los beneficios de implementar la ISO 9001 en diferentes países y contextos socio-económicos como Hong Kong, EEUU, Malaysia, The Netherlands, Hong Kong, España, Reino Unido, Irlanda y Egipto, se puede deducir que los resultados de este trabajo son consistentes con los obtenidos en ellos ${ }^{10}$.

Prosiguiendo con el análisis, se ha profundizado en si las características de los establecimientos hoteleros, como son el tamaño, la categoría del establecimiento, subsector de pertenencia (Hoteles, Balnearios y Paradores) ${ }^{11}$, las certificaciones que poseen, y según la antigüedad en la certificación " $Q$ de Calidad Turística", influyen en los beneficios percibi-

10 Los resultados de estos estudios sugieren que implementar la ISO supone mejoras en la documentación, mejoras en la eficiencia del sistema de calidad, el uso de la ISO como una herramienta promocional, mejora en la calidad de los productos o servicios, mejor selección de los proveedores, mejora de la imagen de la compañía, entre otros, el orden de los factores varia de unos estudios a otros.

11 Por las características diferenciadas de los establecimientos de alojamiento analizados se ha decidido observar las diferencias entre hoteles, balnearios y Paradores. En lo que respecta a los establecimientos termales/balnearios, en los mismos existen dos instalaciones y servicios diferenciados, por un lado el balneario donde se presta el tratamiento termal y por otro, el hotel donde se aloja el termalista. ANBAL (Asociación Nacional de Estaciones Termales, 2006) define el balneario o estación termal como "aquella instalación que dispone de aguas minero-medicinales declaradas de utilidad pública, servicio médico e instalaciones adecuadas para llevar a cabo los tratamientos que se prescriban". Por otro lado, los Paradores de Turismo en España se constituyen como una sociedad anónima de capital público, y engloba bajo su marca un conjunto de hoteles de alta categoría distribuidos por toda España, localizados en edificios emblemáticos o emplazamientos destacables que han sido seleccionados por su interés histórico, artístico o cultural. 
dos. En primer lugar, se realiza la prueba de normalidad de los datos observando que existe ausencia de normalidad $($ sig. $<0,05)$ y se comprobó la igualdad de varianzas mediante el estadístico Levene, los dos supuestos fundamentales que deben cumplirse para poder utilizar la $T$ de Student o ANOVA. En cuanto a la prueba de Levene para igualdad de varianzas en los casos que se observe que las variables no cumplan con la homogeneidad de varianzas se aplicará la prueba no paramétrica Kruskal-Wallis, que proporciona la significación de la Chi-cuadrado, para el resto de los casos se aplicará la T de Student (2 muestras) o Anova (más de dos muestras).

En los casos en que se observen diferencias significativas pero no entre que par de variables al existir más de dos muestras, se utilizará el procedimiento de Scheffé o Games-Howell (prueba no paramétrica) para los casos en que no se cumple los criterios de normalidad y homocedasticida. El Cuadro 2 recoge los resultados del análisis realizado.

Una vez realizado un análisis exploratorio de los beneficios, y con la finalidad de dar cumplimiento al objetivo principal de esta investigación es necesario agrupar a las empresas según los beneficios obtenidos por estas tras su proceso de implantación y certificación de la marca " $Q$ de Calidad Turística", para ello se aplicará el análisis clúster, para analizar su perfil y corroborar las hipótesis planteadas.

Como paso previo al análisis clúster se realizó un análisis factorial exploratorio de componentes principales sobre los datos con la intención de agrupar los 15 beneficios en otras variables que sustituyan a estas con la menor perdida de información posible. Basados en la revisión bibliográfica sobre el tema, se realizó el análisis siguiendo el criterio de "A Priori", considerando sólo dos factores beneficios internos y externos (Tsiotras y Gotzamzni, 1996 y Vloeberhgs y Bellens, 1996).

En primer lugar se comprueba que se puede llevar a cabo el análisis factorial exploratorio, examinando la matriz de correlación, la determinante de la matriz de correlaciones, el test de esfericidad de Bartlett, la medida de adecuación muestral, el índice KMO y la bondad del ajuste del modelo (Tabla 2).

Se comprobó que la matriz de datos es adecuada para su posterior análisis factorial de componentes principales porque cumplen todos los mínimos exigidos en cada uno de los parámetros analizados. Se rotó la matriz con el método varimax para facilitar su interpretación, eliminando de su representación aquellas cargas factoriales con un valor inferior a 0,4 mínimo considerado (Tabla 3). La varianza es de $57,207 \%$ beneficios internos y $77,984 \%$ para los beneficios externos, la cual supera el mínimo exigido del $50 \%$, el Alfa de Cronbach que mide la fiabilidad de la escala es superior a 0,8 mínimo recomendado.

Una vez obtenidos los resultados, mediante este método se han identificado tres factores: 1) Factor 1, denominado "Beneficios internos", está constituido por mejoras de las relaciones dirección empleado, mejora en el ambiente de trabajo, mayor participación de los trabajadores en gestión, incremento de la motivación y satisfacción de los empleados, clara definición de procesos y responsabilidades, 


\section{Cuadro 2 \\ Análisis de la influencia de las características de los establecimientos hoteleros en los beneficios percibidos}

$\begin{gathered}\text { Características de los } \\ \text { establecimientos hoteleros }\end{gathered}$
(beneficios que muestran diferencias signi-
ficativas, sig. $<0,05$ ), lo que implica que
existe asociación entre los beneficios y la
característica correspondiente
Tamaño ${ }^{12}$
a) $\mathbf{N}^{\circ}$ empleados (0-49, pequeña; $50-249$,
mediana y $\geq 250$, grande)
(BF1) Mejora la satisfacción del cliente
(BF6) Mayor participación de los trabajado-
res en gestión
(BF7) Mejora relaciones dirección/empleado
b) $\mathbf{N}^{\circ}$ habitaciones ( $\leq 100$ habitaciones
pequeñas; $>100$ y $\leq 300$ habitaciones me-
dianas; >300 habitaciones grandes)
(BF6) Mayor participación de los trabajado-
res en gestión
(BF7) Mejora relaciones dirección/emplea-
do
(BF9) Productividad/ mejor aprovecha-
miento de tiempo y recursos

Subsector

(BF14) Cuota de mercado

(BF15) Posición competitiva

\begin{abstract}
Importancia de los beneficios
En general las empresas medianas perciben en mayor medida la mejora de la satisfacción del cliente, la mayor participación de los trabajadores y la mejora de las relaciones dirección/empleado.
\end{abstract}

\begin{abstract}
Test de Scheffé
- BF6 el test no mostró diferencias significativas, por lo que no se puede asegurar que existan diferencias significativas al nivel significativo de 0,05 .

- BF7 y BF9 el test de Games-Howell mostró diferencias significativas:

- Las empresas de menos de 100 habitaciones perciben en menor medida que las relaciones dirección/empleado mejoren.

- Las empresas de menos de 100 habitaciones tienen una percepción menor de que haya mejorado la productividad, obteniendo un menor aprovechamiento de tiempo y recursos, que las empresas de entre 100 y 300 habitaciones al tener documentados los procesos.

- Observando también que la mejora de las relaciones dirección/empleado es proporcional al número de habitaciones en los tres grupos analizados.

Importancia de los beneficios

Las empresas grandes perciben en mayor medida la mayor participación de los trabajadores en gestión, la mejora de las relaciones dirección/empleado y el incremento de la productividad.
\end{abstract}

\section{Test de Scheffé}

- BF14 y BF15 el test no mostró diferencias significativas, por lo que no se puede asegurar que existan diferencias significativas al nivel significativo de 0,05 .

Importancia de los beneficios

Las diferencias se dan entre los hoteles de 1-2 estrellas (*) y los de 4-5 estrellas $\left(^{*}\right)$ en el sentido de que los de menor categoría han percibido un menor incremento de las ventas y los hoteles de mayor categoría han percibido un mayor incremento en su cuota de mercado.

12 El tamaño del hotel puede medirse por su número de habitaciones sobre huéspedes, aunque el número de investigadores que así lo hacen son pocos. En este sentido, Vallen y Vallen (1991) consideran a un hotel pequeño cuanto tiene 100 o menos habitaciones, mediano entre 100 y 300, y grande con más de 300 habitaciones. Por otra parte, investigadores como Lattin (1994), Muñoz Oñate (1994), y Renner (1994) en sus investigaciones diferencian entre pequeños, medianos y grandes, pero no delimitan el número de habitaciones que corresponden a cada grupo. 


\section{Cuadro 2 (Continuación)}

\begin{tabular}{|c|c|}
\hline $\begin{array}{l}\text { Características de los } \\
\text { establecimientos hoteleros } \\
\text { (beneficios que muestran diferencias signi- } \\
\text { ficativas, sig. }<0,05 \text { ), lo que implica que } \\
\text { existe asociación entre los beneficios y la } \\
\text { característica correspondiente }\end{array}$ & Análisis \\
\hline $\begin{array}{l}\text { Categoría (1-2, 3, 4-5 estrellas }{ }^{13} \text { ) } \\
\text { (BF13) Ventas } \\
\text { (BF14) Cuota de mercado } \\
\text { (BF15) Posición competitiva }\end{array}$ & $\begin{array}{l}\text { Test de Scheffé } \\
\text { - Las diferencias se dan entre los hoteles de 1-2 estrellas * y los de 4-5 estre- } \\
\text { llas * en el sentido de que los de menor categoría han percibido un menor } \\
\text { incremento de las ventas y los hoteles de mayor categoría han percibido un } \\
\text { mayor incremento en su cuota de mercado. } \\
\text { - El test de Scheffé no permite asegurar que existan diferencias significativas al } \\
\text { nivel significativo de 0,05 en el caso de la posición competitiva. } \\
\text { Importancia de los beneficios } \\
\text { En general las empresas con una clasificación en la categoría mayor, perci- } \\
\text { ben que la implantación de su Sistema de Gestión de la Calidad les ha permi- } \\
\text { tido en mayor medida que al resto de las categorías incrementar las ventas, } \\
\text { su cuota de mercado y su posición competitiva. }\end{array}$ \\
\hline
\end{tabular}

Certificaciones que poseen*

Encontrándose diferencias en 11 de los 15 beneficios analizados (no se encuentran diferencias en BF4, BF9,BF10,BF11)

Según la antigüedad en la certificación (> 5 años; $\leq 5$ años)

(BF7) mejoran las relaciones dirección/empleado

\section{Importancia de los beneficios}

En general las empresas con alguna otra certificación a mayores de la "Q" perciben en mayor medida los beneficios analizados.

\section{Importancia de los beneficios}

En general las empresas con mayor antigüedad en la certificación perciben en mayor medida que mejoran las relaciones dirección/empleado.

* Están certificadas únicamente en la "Q de Calidad Turística" o tienen certificado otro SGC como la ISO 9001 o la ISO 14001- "Sistema de Gestión Medioambiental".

Fuente: Elaboración propia.

incremento de la conciencia de calidad de los empleados, mejor aprovechamiento del tiempo y recursos y reducción de costos; 2) Factor 2, "Beneficios externosclientes", constituido por mejor conocimiento de las expectativas del cliente, servicios de calidad, reducción de quejas y mejora la satisfacción del cliente, y Fac- tor 3, "Beneficios externos- financieros", mejoras en la cuota de mercado, ventas y posición competitiva.

Para finalizar, se realiza el análisis del impacto de la implantación y posterior certificación en los beneficios considerados (Tabla 4). Existe un mayor impacto en los beneficios internos con una media

13 En España el RD 1.634/1983 de 15 de junio, clasifica los hoteles y hoteles-apartamentos en cinco categorías, identificadas por estrellas. En dicho decreto se dispone que la determinación de las categorías de los hoteles se hará en virtud del cumplimiento de unos requisitos técnicos mínimos agrupados en cinco apartados: instalaciones, comunicaciones, zona de clientes, servicios generales y zona de personal. 


\section{Tabla 2}

\section{Indicadores del grado de asociación entre variables beneficios internos* $y$ externos}

\begin{tabular}{|c|c|c|c|c|c|c|}
\hline$\frac{\text { Indicador }}{\text { Escala }}$ & $\begin{array}{c}\text { Matriz de } \\
\text { correlaciones }\end{array}$ & $\begin{array}{l}\text { Determinante } \\
\text { de la matriz de } \\
\text { correlaciones }\end{array}$ & $\begin{array}{l}\text { Test de } \\
\text { esfericidad } \\
\text { de Bartlett }\end{array}$ & $\begin{array}{c}\text { Medida de } \\
\text { adecuación } \\
\text { de la muestra }\end{array}$ & $\begin{array}{l}\text { Índice de } \\
\text { KMO }\end{array}$ & $\begin{array}{l}\text { Bondad del } \\
\text { ajuste del } \\
\text { modelo }\end{array}$ \\
\hline $\begin{array}{l}\text { Beneficios } \\
\text { internos }\end{array}$ & $\begin{array}{l}\text { Variables } \\
\text { correlacionadas }\end{array}$ & 0,010 & $\begin{array}{c}836,563 \text { sig. } \\
0,000\end{array}$ & $(0,942-0,856)$ & 0,852 & $46 \%$ \\
\hline $\begin{array}{l}\text { Beneficios } \\
\text { externos }\end{array}$ & $\begin{array}{l}\text { Variables } \\
\text { correlacionadas }\end{array}$ & 0,006 & $\begin{array}{c}927,759 \text { sig. } \\
0,000\end{array}$ & $(0,851-0,888)$ & 0,838 & $33 \%$ \\
\hline
\end{tabular}

* El Test de esfericidad de Bartlett muestra que la Chi-cuadrado aproximado es muy alta y con un nivel de significación inferior a 0,05 máximo permitido, y la medida de adecuación muestral Kaiser-Meyer-Oklin es superior al 0,05 mínimo permitido. Por último, la bondad del ajuste del modelo es inferior al $50 \%$ permitido con valores absolutos mayores que 0,05 de residuos no redundantes cumpliéndose todos los criterios analizados. Todos los parámetros analizados nos indican que es factible proseguir con el análisis factorial.

Fuente: Elaboración propia.

de 5,7520 siendo prácticamente igual la importancia con los beneficios externos con una media de 5,6912. Por otro lado si se analizan, beneficios internos, beneficios externos-clientes, y beneficios externos-financieros, los más importantes son los externos de clientes y los menos importantes son los externos financieros.

Prosiguiendo, se lleva a cabo la agrupación de las empresas mediante el análisis Clúster, utilizando las puntuaciones factoriales de las variables y se aplica en primer lugar el análisis jerárquico (método de Ward para minimizar las diferencias dentro de los conglomerados y la distancia Euclídea al cuadrado), con el objetivo de identificar el número apropiado de grupos o conglomerados (Tabla 5). Para ello se observa el dendograma y el coeficiente de aglomeración.
Se observa que con respecto a los coeficientes de aglomeración los cambios más importantes se producen entre 2 y 4 , y utilizando el criterio de las diferencias entre los cambios porcentuales, se elegiría aquel donde sea mayor la diferencia entre cada par de cambios, en este caso en cuatro grupos $(32,0573)$, siendo el número ideal de grupos según el coeficiente de aglomeración es de cuatro grupos. Se procede a continuación a realizar el análisis k- medias, utilizando el número de grupos obtenidos y tomando como centros iniciales los resultados del análisis jerárquico (medias de las puntuaciones factoriales de los cuatro grupos). Para finalizar, se valida los resultados con el análisis de la varianza de un factor $^{14}$ y se comprueba que son significativos los tres factores; beneficios inter-

14 La Fuente, Salas y Pérez (1985:142) consideran este criterio estadístico, F de Fisher, para determinar el número de grupos. 


\section{Matriz rotada de los beneficios para certificarse}

\begin{tabular}{|c|c|c|c|c|c|}
\hline & Beneficios & $\begin{array}{l}\text { Revisión } \\
\text { bibliográfica } \\
\text { Clase }\end{array}$ & $\begin{array}{l}\text { Factor } 1 \\
\text { Interna }\end{array}$ & $\begin{array}{l}\text { Factor } 2 \\
\text { Externa }\end{array}$ & $\begin{array}{l}\text { Factor } 3 \\
\text { Externa }\end{array}$ \\
\hline (BF7) & Mejora las relaciones dirección empleado & Interna & 0,807 & & \\
\hline (BF3) & Mejora en el ambiente de trabajo & Interna & 0,872 & & \\
\hline (BF6) & $\begin{array}{l}\text { Mayor participación de los trabajadores } \\
\text { en gestión }\end{array}$ & Interna & 0,799 & & \\
\hline (BF4) & $\begin{array}{l}\text { Incremento de la motivación/ satisfacción } \\
\text { de los empleados }\end{array}$ & Interna & 0,826 & & \\
\hline (BF2) & $\begin{array}{l}\text { Clara definición de procesos/responsabilida- } \\
\text { des }\end{array}$ & Interna & 0,671 & & \\
\hline (BF8) & $\begin{array}{l}\text { Incremento de la conciencia de calidad } \\
\text { de los empleados }\end{array}$ & Interna & 0,653 & & \\
\hline (BF9) & $\begin{array}{l}\text { Productividad/ mejor aprovechamiento } \\
\text { de tiempo y recursos }\end{array}$ & Interna & 0,743 & & \\
\hline (BF11) & Reducción de Costos & Interna & 0,645 & & \\
\hline (BF5) & $\begin{array}{l}\text { Mejor conocimiento de las expectativas } \\
\text { del cliente }\end{array}$ & Externa & & 0,883 & \\
\hline (BF12) & Servicios de calidad & Externa & & 0,673 & \\
\hline (BF10) & Reducción de quejas & Externa & & 0,760 & \\
\hline (BF1) & Mejora la satisfacción del cliente & Externa & & 0,819 & \\
\hline (BF14) & Cuota de mercado & Externa & & & 0,921 \\
\hline (BF13) & Ventas & Externa & & & 0,917 \\
\hline (BF15) & Posición competitiva & Externa & & & 0,876 \\
\hline \multicolumn{2}{|c|}{ Valor propio } & & 4,577 & 2,768 & 2,690 \\
\hline \multicolumn{2}{|c|}{$\%$ de la varianza explicada por factor } & & 57,207 & 39,550 & 38,435 \\
\hline \multicolumn{2}{|c|}{$\%$ acumulado de varianza explicada } & & 57,207 & \multicolumn{2}{|c|}{77,984} \\
\hline \multicolumn{3}{|c|}{ Alfa de Cronbach estandarizado } & 0,887 & \multicolumn{2}{|c|}{0,891} \\
\hline
\end{tabular}

Fuente: Elaboración propia.

Tabla 4

Importancia de los beneficios después de certificarse

\begin{tabular}{lcc}
\hline \multicolumn{1}{c}{ Factores } & Media (de 1 a 7) & Desviación típica \\
\hline Beneficios internos & 5,7520 & 0,74383 \\
Beneficios externos $^{15}$ & 5,6912 & 0,81052 \\
Beneficios externos-clientes $_{\text {Beneficios externos-financieros }}$ & 5,9140 & 0,82076 \\
\hline
\end{tabular}

Fuente: Elaboración propia.

15 Esta variable agrupa a las razones externas de mercado y de exigencias con el objeto de establecer comparaciones con otros trabajos revisados previamente. 
Tabla 5

Coeficiente de aglomeración de los beneficios derivados de la implantación y certificación con la marca " $Q$ de calidad Turística"

\begin{tabular}{cccc}
\hline $\mathbf{N}^{\circ}$ de grupos & $\begin{array}{c}\text { Coeficiente de } \\
\text { aglomeración }\end{array}$ & $\begin{array}{c}\text { Cambio porcentual } \\
\text { del coeficiente }\end{array}$ & $\begin{array}{c}\text { Diferencias entre los } \\
\text { cambios porcentuales }\end{array}$ \\
\hline 10 & 102,316 & 13,4026 & $-1,0514$ \\
9 & 116,029 & 12,3511 & $-1,0221$ \\
8 & 130,360 & 11,3290 & 2,3491 \\
7 & 145,128 & 13,6781 & $-0,9833$ \\
6 & 164,979 & 12,6947 & 0,5868 \\
5 & 185,923 & 13,2815 & 0,6055 \\
4 & 210,616 & 13,8870 & 32,0573 \\
3 & 239,865 & 45,9443 & 12,5959 \\
2 & 350,069 & 58,5402 & \\
1 & 555,000 & & \\
\hline
\end{tabular}

Fuente: Elaboración propia.

nos (F-85,413; sig. 0,000), beneficios externos clientes (F-112,003; sig. 0,000) y beneficios externos financieros (F117,227; sig. 0,000).

El siguiente paso es la interpretación de los cuatro grupos creados para determinar si existen diferencias entre ellos. En la Tabla 6 aparecen reflejadas las puntuaciones medias de las variables originales ${ }^{16}$ que se incluyen en cada factor para cada grupo (en lugar de las puntuaciones factoriales) para determinar los perfiles medios.

El primer grupo está formado por 75 empresas que percibieron altos beneficios tanto internos como externos (clientes y financieros). El segundo grupo está formado por 46 empresas con una valoración baja de los beneficios tanto internos como externos, y el grupo 3 lo constituyen las empresas que obtuvieron beneficios altos internos y externos de clientes, pero percibieron bajos beneficios financieros. El último lo constituyen aquellas empresas que obtuvieron beneficios medios tanto internos como externos-clientes y beneficios financieros bajos. Como conclusión final se puede afirmar que 150 empresas obtuvieron beneficios internos y externos-clientes altos o medios. Por el contrario 111 empresas percibieron beneficios externos financieros bajos, de esta forma se corroboran los resultados obtenidos en el análisis descriptivo.

Para finalizar se aplica la prueba Chi-cuadrado con la finalidad de comprobar si existen diferencias significativas

16 Para calcular la media por grupo en cada factor se incluyeron las variables con mayor carga. 
Tabla 6

Medias por factor y pruebas estadísticas para comparar diferencias

\begin{tabular}{lcccccc}
\hline \multirow{2}{*}{ Factores } & \multicolumn{3}{c}{ Medias* $^{*}$} & \multicolumn{2}{c}{ Kruskal-Wallis** $^{*}$} \\
\cline { 2 - 7 } & $\begin{array}{c}\text { Grupo 1 } \\
\mathbf{n = 7 5}\end{array}$ & $\begin{array}{c}\text { Grupo 2 } \\
\mathbf{n = 4 6}\end{array}$ & $\begin{array}{c}\text { Grupo 3 } \\
\text { n=22 }\end{array}$ & $\begin{array}{c}\text { Grupo 4 } \\
\text { n=43 }\end{array}$ & Chi- cuadrado & Sig. \\
Beneficios internos & 6,2983 & 4,8478 & 5,9148 & 5,6831 & 110,194 & 0,000 \\
Beneficios externos clientes & 6,5333 & 4,8859 & 6,3295 & 5,7209 & 119,766 & 0,000 \\
Beneficios externos financieros & 6,3956 & 4,9130 & 4,1061 & 4,8217 & 133,459 & 0,000 \\
\hline
\end{tabular}

* Aparecen reflejadas las puntuaciones medias de las variables originales que se incluyen en cada factor para cada grupo (en lugar de las puntuaciones factoriales) para determinar los perfiles medios. Por otro lado, se realiza la prueba de Kruskal-Wallis para analizar las diferencias de medias. Optamos por utilizar esta prueba en lugar de a Anova puesto que al dividir la muestra en cuatro conglomerados, el número de empresas perteneciente a cada grupo es pequeño, por lo que se considera más conveniente aplicar la prueba $\mathrm{H}$ de Kruskal-Wallis.

** Optamos por utilizar la prueba de Kruskal-Wallis para analizar las diferencias de medias, en lugar de Anova puesto que al dividir la muestra en cuatro conglomerados, el número de empresas perteneciente a cada grupo es pequeño, por lo que se considera más conveniente aplicar esta prueba.

Fuente: Elaboración propia.

entre los grupos dependiendo del tamaño de las empresas ${ }^{17}$ y el subsector de pertenencia (Tabla 7). Los datos reflejan que existen diferencias significativas entre los grupos en relación a las variables tamaño y subsector, por lo que queda demostrado los beneficios percibidos por las empresas tras la implementación y certificación dependen de si la empresa es de pequeño o mediano y del subsector de pertenencia (Hotel, Balneario y Parador).

Al análisis clúster realizado, es decir, al perfil de las empresas se añaden variables adicionales (factores críticos y resultados) lo que permitirá contrastar las hipótesis planteadas. Los datos de las variables adicionales forman parte de otro apartado del trabajo de investigación de- sarrollado en el que se analiza además de los beneficios, los factores críticos de la calidad y sus resultados. Se consideran como factores críticos: política de calidad (7 ítems), liderazgo (8), alianzas y recursos (7), aprendizaje (9), gestión de empleados (11), gestión de procesos (15) y, como resultados: satisfacción de los clientes (7), satisfacción empleados (9), resultados impacto social (8), resultados clave (12). El análisis factorial realizado sobre los factores críticos ha permitido considerar de manera agregada a los mismos ${ }^{18}$.

Según la revisión bibliográfica revisada el hecho de implantar un sistema de calidad tiene efectos positivos para la empresa (Bulled, 1987), por ello se supone

17 La clasificación en microempresas, pequeñas, medianas y grandes empresas se ha realizado en base al criterio del número de trabajadores según la recomendación de la Comisión de las Comunidades Europeas 96/280/CE, de 3 de abril de 1996, sobre la definición de pequeñas y medianas empresas (Diario Oficial $n^{\circ}$. L107 de 30/04/1996, pp. 4-9). Nuestra muestra está formada por empresas de pequeño y mediano tamaño. 
Tabla 7

Porcentaje de empresas pertenecientes a cada grupo según el tamaño y subsector y la prueba Chi-cuadrado

\begin{tabular}{lcccccc}
\hline & $\begin{array}{c}\text { Grupo 1 } \\
\mathbf{n = 7 5}\end{array}$ & $\begin{array}{c}\text { Grupo 2 } \\
\mathbf{n = 4 6}\end{array}$ & $\begin{array}{c}\text { Grupo 3 } \\
\mathbf{n = 2 2}\end{array}$ & $\begin{array}{c}\text { Grupo 4 } \\
\mathbf{n = 4 3}\end{array}$ & Chi-cuadrado & Sig. \\
\hline Pequeña & 54,7 & 71,7 & 40,9 & 39,5 & & \\
Mediana & 45,3 & 28,3 & 59,1 & 60,5 & 10,968 & 0,012 \\
Total & 100,0 & 100,0 & 100,0 & 100,0 & & \\
Hotel & 69,3 & 67,4 & 90,9 & 90,7 & & \\
Balneario & 10,7 & 6,5 & 0,0 & 4,7 & $12,970^{*}$ & 0,031 \\
Parador & 20,0 & 26,1 & 9,1 & 4,7 & & \\
Total & 100,0 & 100,0 & 100,0 & 100,0 & & \\
\hline
\end{tabular}

* Valor estadístico de Fisher y la significación exacta asociada porque existen más de un veinticinco por ciento de casillas con frecuencia esperada inferior a cinco.

Fuente: Elaboración propia.

que cuanto mayores sean los beneficios conseguidos gracias a la misma, la empresa implementará mayores niveles de calidad. Por ello se plantean las siguientes hipótesis finales:

H1: Cuanto mayores sean los niveles de beneficio derivados de la implementación y certificación mayor será el nivel de implementación de los factores críticos de la calidad.

H2: Cuanto mayores sean los niveles de beneficios derivados de la implementación y certificación mayor serán los resultados obtenidos por la empresa.
Para ello en primer lugar se analiza la matriz de correlaciones (Tabla 8).

Se observa que en todos los casos existe correlación excepto en resultados en la sociedad. Con lo que queda demostrado que los elementos de la calidad están relacionados con los beneficios. En la Tabla 9 se puede observar que los resultados obtenidos permiten corroborar las dos hipótesis planteadas.

En el grupo 1 que es el más numeroso se observa que los beneficios que le reportó la implantación y posterior certificación son altos, lo que ha influido en un mayor nivel de implantación de los facto-

18 Tarí (2000) considera de manera agregada los seis factores críticos de la calidad: (1) Factores humanos (Liderazgo y Aprendizaje); (2) Factores técnicos (Política/Planificación de la calidad, Alianzas y recursos, Gestión de empleados, Mejora continua y gestión de procesos). En otros estudios, Beltrán et al. (2003) agrupa los elementos de la calidad en Elementos sociales o "soft" de la calidad (Personas y Liderazgo que recogen la vertiente humana y social de la GCT y Elementos técnicos o "hard" de la calidad (Procesos y Alianzas) que recogen una orientación operativa. El criterio Política/Planificación de la calidad consideran que actúa como guía para la gestión del resto de los elementos (Reiner, 2002). 


\section{Tabla 8}

Correlación de Pearson entre los beneficios, los factores críticos y resultados

\begin{tabular}{llccccc}
\hline & & $\begin{array}{c}\text { Factores } \\
\text { críticos }\end{array}$ & $\begin{array}{c}\text { Resultados } \\
\text { en los } \\
\text { clientes }\end{array}$ & $\begin{array}{c}\text { Resultados } \\
\text { en los } \\
\text { empleados }\end{array}$ & $\begin{array}{c}\text { Resultados } \\
\text { en la } \\
\text { sociedad }\end{array}$ & $\begin{array}{c}\text { Resultados } \\
\text { clave }\end{array}$ \\
\hline Beneficios & $r$ & $0,422^{* *}$ & $0,338^{* *}$ & $0,390^{* *}$ & 0,140 & $0,252^{* *}$ \\
internos & Sig. & 0,000 & 0,000 & 0,000 & 0,058 & 0,001 \\
Beneficios & $r$ & $0,263^{* *}$ & $0,320^{* *}$ & $0,252^{* *}$ & 0,080 & $0,226^{* *}$ \\
externos de clientes & Sig. & 0,000 & 0,000 & 0,001 & 0,279 & 0,002 \\
Beneficios & $r$ & $0,319^{* *}$ & $0,299^{* *}$ & $0,313^{* *}$ & $0,319^{* *}$ & $0,235^{* *}$ \\
externos financieros & Sig. & 0,000 & 0,000 & 0,000 & 0,000 & 0,001 \\
\hline
\end{tabular}

$r=$ correlación de Pearson.

**La correlación es significativa al 0,01.

Fuente: Elaboración propia.

Tabla 9

Medias por factor y pruebas estadísticas para comparar diferencias

\begin{tabular}{lrrrrrrr}
\hline \multicolumn{1}{c}{ Factores } & \multicolumn{4}{c}{ Medias } & \multicolumn{2}{c}{ Kruskal-Wallis* } \\
\cline { 2 - 7 } & Grupo 1 & Grupo 2 & Grupo 3 & Grupo 4 & Chi- cuadrado & Sig. \\
& $\mathbf{n = 7 5}$ & $\mathbf{n = 4 6}$ & $\mathbf{n = 2 2}$ & $\mathbf{n = 4 3}$ & & \\
\hline Beneficios internos & 6,2983 & 4,8478 & 5,9148 & 5,6831 & 110,194 & 0,000 \\
Beneficios externos clientes & 6,5333 & 4,8859 & 6,3295 & 5,7209 & 119,766 & 0,000 \\
Beneficios externos financieros & 6,3956 & 4,9130 & 4,1061 & 4,8217 & 133,459 & 0,000 \\
Factores críticos & 6,2578 & 5,5652 & 6,2073 & 5,6675 & 31,144 & 0,000 \\
Resultados en los clientes & 6,6552 & 6,2205 & 6,5909 & 6,3821 & 23,558 & 0,000 \\
Resultados en los empleados & 5,4296 & 4,7657 & 5,2020 & 5,2326 & 11,501 & 0,009 \\
Resultados en la sociedad & 5,5000 & 5,0679 & 4,1250 & 5,2384 & 14,616 & 0,002 \\
Resultados clave & 5,0109 & 4,4763 & 4,6529 & 4,6321 & 17,428 & 0,001 \\
\hline
\end{tabular}

* Se utiliza esta prueba puesto que es la adecuada en este caso dada la ausencia de normalidad de las variables utilizadas en el análisis. Se trata de la versión no paramétrica de la T-Student, obteniéndose diferencias significativas entre diferentes grupos con relación a una serie de variables.

Fuente: Elaboración propia. 
res críticos. Del mismo modo, en el grupo dos donde los beneficios percibidos son bajos también el nivel de implantación de los factores críticos es bajo. Ello permite corroborar la hipótesis H1. En este mismo sentido, en el grupo tres se observa que cuando son altos los beneficios externos de los clientes ello repercute en un mayor nivel de los factores críticos. En lo que respecta a la hipótesis $\mathrm{H} 2$, esta se confirma puesto que en el grupo 1 y 3 donde los beneficios percibidos son altos, también son más altos los resultados obtenidos.

\section{Conclusiones}

En primer lugar se destaca que la novedad del estudio radica en dos puntos: 1) se lleva a cabo en empresas de alojamiento turístico, sector donde no existen prácticamente estudios que analicen la implantación de sistemas de gestión de calidad y sus consecuencias en el funcionamiento de la empresa; y 2) los alojamientos analizados poseen un sistema de gestión de la calidad basado en normas UNE 182001:2008 (marca Q de calidad turística), lo que permite comparar los resultados de otros estudios realizados en empresas con una implantación en base a la norma ISO 9001, con los resultados obtenidos con un SGC específico del sector turístico como es la $Q$ de Calidad Turística.

Así, la experiencia de las empresas de alojamiento turístico que tienen implantada la Marca Q de Calidad Turística puede servir de orientación para aquellas otras que están planteándose implementar la norma o ya la tengan implementada. De esta forma, los gerentes al conocer y entender los beneficios que pueden obtener de la implantación supondrá un impulso del sector hacia la implantación de un SGC o el avance hacia la mejora continua para todas aquellas que ya lo tengan implantado alcanzando una mayor eficiencia en el proceso de implantación y de esta forma incrementar sus posibilidades de éxito.

Los resultados muestran que las empresas participantes en esta investigación perciben que implantar un SGC:

- Les ha ayudado a conocer en mayor profundidad los procedimientos así como a fijar las responsabilidades dentro de la organización, lo que les proporciona una mayor seguridad en lo que se hace y repercute en una mejor atención a los clientes, en la mejora de los servicios que se ofrece, y por supuesto, en un importante ahorro de costos.

- Ha incrementado la conciencia de calidad de los empleados, lo que permitirá mejorar la calidad del servicio interno, de modo que esta repercuta sobre la satisfacción del cliente externo $y$, por tanto, en el desempeño organizativo.

- Tienen un mejor conocimiento de las expectativas del cliente, es decir, los deseos de los consumidores. Se debe tener en cuenta que las empresas deben aprender a gestionar las expectativas de los clientes, puesto que, la calidad de servicio es un concepto relativo, que viene determinado por la diferencia existente entre las percepciones y expectativas que este tiene y el nivel al cual la empresa consigue satisfacerlas.

Por el contrario, los beneficios menos valorados han sido la reducción de costos y el incremento de las ventas y cuo- 
ta de mercado. En general podemos afirmar basándonos en los datos que todos los beneficios analizados han sido muy bien valorados, lo que lleva a plantearse que son muchos y muy importantes los beneficios derivados de la implantación de un SGC, ya que repercute en aspectos internos y supone mejoras significativas en su rendimiento o en el grado de satisfacción e integración de los trabajadores.

En esta investigación, los datos muestran que existen diferencias significativas entre los beneficios percibidos por las empresas y el tamaño, el subsector de pertenencia, la categoría y las certificaciones que poseen. No se ha profundizado en cuáles son las razones que explican estos hechos pero si se considera necesario, a través de una investigación cualitativa, entrevistas en profundidad, que en posteriores estudios se llevará a cabo.

En lo referente a la estructura de los beneficios percibidos por las empresas en el sector de alojamiento turístico, se ha llegado a la conclusión de que están agrupados en tres grupos: beneficios internos, beneficios externos de clientes $y$ beneficios externos-financieros y se observa que las empresas consideran igual de importantes los beneficios internos y externos (los más importantes son los externos de clientes y los menos son los beneficios financieros).

Para finalizar, se ha corroborado que cuanto mayor sean los niveles de beneficios derivados de la implantación y certificación mayores serán los niveles de implementación de los factores críticos. Lo que supone que la obtención de beneficios derivados de la implementación de la calidad favorece e impulsa el proceso de implementación de los factores críticos de la calidad (política de calidad, liderazgo, alianzas y recursos, aprendizaje, gestión de empleados, gestión de procesos, mejora continua). También se corroboró que cuanto mayores sean los niveles de beneficios derivados de la implantación y certificación serán también mayores los resultados obtenidos por la empresa, lo que supondrá la obtención de unos mayores resultados en los clientes, empleados, en la sociedad y en los resultados clave, al existir un mayor desarrollo de los factores críticos. En este sentido un elevado compromiso de los directivos con la estrategia de mejora de la calidad, que se evidencia en su participación activa en el despliegue y revisión de la misma, sirviendo su comportamiento de referencia tanto para su personal como antes otros grupos de interés de la empresa, redunda en la obtención de unos mejores resultados.

\section{Referencias Bibliográficas}

Aarts, Frank Martin y Vos, Ed (2001). The impact of ISO registration on New Zealand firms performance: A financial perspective. The TQM Magazine, Vol. 13, No. 3, pp. 180-191.

Adanur, Sabit y Allen, Brian (1995). First results on the effects of ISO 9000 in the US textile industry. Benchmarking for Quality Management \& Technology, Vol. 2, No. 3, pp. 41-52.

Asociación Nacional de Estaciones Termales ANET/Asociación Nacional de Balnearios - ANBAL. Información datos del termalismo en España Mensaje recibida por anet@balnearios.org en 14 jun. 2006.

Alonso-Almeida, María del Mar, Rodríguez-Antón, José Miguel; Rubio-Andrada, Luis 
(2012). Reasons for implementing certified quality systems and impact on performance: an analyses of the hotel industry. The Service Industries Journal, Vol. 32, pp. 919-936.

Álvarez, José; Fraiz, José Antonio; Del Río, María de la Cruz (2011). The Q for Tourist Quality barriers found in the implementation process by certified Spanish resorts. International Conference "From Villes Thermales To Sustainable Health And Wellness Destinations", Chaves.

Babbie, Earl (1995). The Practice of Social Research. Wadsworth Publishing Company.

Benner, Mary J. and Veloso, Francisco M. (2008). ISO 9000 Practices and Financial Performance: A Technology Coherence Perspective. Journal of Operations Management, Vol. 26, No. 5, pp. 611-629.

Brecka, Jon (1994). Study finds gains with ISO 9000 registration increase over time. Quality Progress, pp. 18-20.

Brown, Alan y Van der Wiele, Ton (1995). Survey on Australian self-assessment. The Quality Magazine, Vol. 5 No. 3, pp. 18-24.

Brown, Alan; Van Der Wiele, Ton y Loughton, Kate (1998). Smaller enterprises experiences with ISO 9000. International Journal of Quality and Reliability Management, Vol. 15, No. 3, pp. 273-285.

Bulled, J.W. (1987). BS 5750-Auality management systems and assessment. General Engineer, november, pp. 271280.

Buttle, Francis (1997). ISO 9000: marketing motivations and benefits. International Journal of Quality \& Reliability Management, Vol. 14, No. 9, pp. 936-947.

Camisón, César y Yepes, Víctor (1994). Normas ISO-9000 y la gestión de calidad total en la empresa turística. Primer
Congreso de Calidad de la Comunidad Valenciana. Libro de ponencias, mayo, pp. 583-620.

Camisón, César (1996). Total Quality Management in hospitality: an application of the EFQM model. Tourism Management, Vol. 17, No. 3, pp191-201.

Camisón, Cesar; Cruz, Sonia y González, Tomás (2007). Gestión de la calidad: conceptos, enfoques y sistemas. Madrid: Pearson - Prentice Hall. Madrid.

Carlsson, Matts; Carlsson, Dan (1996). Experiences of implementing ISO 9000 in Swedish industry. International Journal of Quality and Reliability Management, Vol. 13, No. 7, pp. 36-47.

Casadesús Fá, Martí y Heras Saizarbitoria, Iñaki (2005). El Boom de la calidad en las empresas españolas. Universia business review, Vol. 7, tercer trimestre, pp. 90-101.

Casadesús Fá, Martí; Heras Saizarbitoria, Iñaki; Arana Landín, Germán (2004). Costos y beneficios de la implantación de la normativa de calidad ISO 9000. Evolución temporal. Congreso nacional de ACEDE, Murcia.

Casadesús Fá, Martí; Gimenez Leal, Gerusa. y Heras Saizarbitoria, Iñaki (2001). Benefits of ISO 9000 implementation in Spanish industry. European Business Review, Vol. 13, No. 6, pp. 327-336.

Casadesús Fá, Martí; Heras Saizarbitoria, Iñaki y Ochoa Laburu, Carlos (2000). Implantación de la normativa ISO 9000 en las comunidades autónomas de Cataluña y el país vasco: conclusiones de dos estudios empíricos. Revista De Dirección y Administración De Empresas, Vol. 8, pp. 43 - 60.

Casadesús Fá, Martí y Karapetrovic, Stanislav (2005). An empirical study of the benefits: a temporal study. International Journal of Quality and Reliability Management, Vol. 16, No. 1, pp. 105-120. 
Casadesús Fá, Martí.; Marimon Viadiu, Frederic. y Alonso, Mar (2010). The future of standardised quality management in tourism: evidence from the Spanish tourist sector". The Service Industries Journal, Vol. 30 , No. 14, pp. 2457-2474.

Dick, Gavin; Gallimore, Kevin; Brown, Jane C. (2001). "ISO 9000 and quality emphasis: an empirical study of front-room versus back-room dominant service industries. International Journal of Service Industry Management, Vol. 12, No.2, pp.114-36.

Dick, Gavin P.M.; Heras, Iñaki. y Casadesús, Martí (2008). Shedding light on causation between ISO 9001 and improved business performance. International Journal of Operations \& Production Management, Vol. 28, No. 7, pp. 687-708.

Dissanayaka, Sunil M.; Kumaraswamy, Moham; Karim, K. y Marosszeky, Marton (2001). "Evaluating outcomes from ISO 9000 certified quality systems of Hong Kong constructors. Total Quality Management, Vol. 12, No. 1, pp. 29-40.

Escanciano, Carmen; Fernández, Esteban; Vázquez, Camilo (2001). ISO 9000 certification and quality management in Spain: Results of a national survey. TQM Magazine, Vol. 13, No. 3, pp. 192-200.

Eskildsen, Jacob K.; Kristensen, Kristensen y Juhl, Hans Jorn (2001). The criterion weights of the EFQM excellence model. International Journal of Quality \& Reliability Management, Vol. 18, No. 8, pp. 783-795.

Ferguson, Wade (1996). Impact of ISO 9000 on industrial marketing. Industrial Marketing Management, Vol. 25, No. 4, pp. 305-310.

Ghobadian, Abby y Gallear, D.N. (1996). Total quality management in SMEs. Omega. The International Journal of Ma- nagement Science, Vol. 24, No. 1, pp. 83-106.

Gotzamani, Katerina D. y Tsiotras, George D. (2002). The true motivations behind ISO 9000 certification: Their effect on the overall certification benefits and long term contribution towards TQM. International Journal of Quality, Vol. 19, No. 2, pp. 151-169.

Grande, Ildefonso y Abascal, Elena. (2000). Fundamentos y Técnicas de Investigación Comercial. $5^{\mathrm{a}}$ edición, ESIC Editorial, Madrid.

Gustafsson, Roberth; Klefsjö, Bengt.; Berggren Eric.; Wellemets Ulrika. Granfors (2001). Experiences from implementing ISO 9000 in small enterprises - a study of Swedish organizations. The TQM Magazine, Vol. 13, No. 4, pp. 232-246.

Hamilton, Robin (1996). Utilizing ISO 9002 and IIP to maximize quality and profitability. Training For Quality, Vol. 4, No. 6, pp. 18-19.

Hardie, Neil (1998). The effects of quality on business performance. Quality Management Journal, Vol. 5, No. 3, pp. 65-83.

Häversjö, Tord (2000). The financial effects of ISO 9000 registration for Danish companies. Managerial Auditing Journal, Vol. 15, No.1.

Hendricks, Kevin B. y Singhal, Vinod R. (1996). Quality awards and the market value of the firm: an empirical investigation. Management Science, Vol. 42, No. 3, pp. 415-436.

Hendricks, Kevin B.; Singhal, Vinod R. (2001a). Firm Characteristic, Total Quality Management, and Financial Performance. Journal of Operations Management, Vol. 19, No. 3, pp. 269-285.

Hendricks, Kevin B.; Singhal, Vinod R. (2001b). The Long-Run Stock Price Performance of Firms with Effective TQM Programs. Management Science, Vol. 47, No. 3, pp. 359-368. 
Heras, Iñaki; Arana, Germán y Casadesús, Martí (2006). The impact of quality management in European companies' performance: the case of the Spanish companies. European Business Review, Vol 18, No. 2, pp. 114-131.

Heras, Iñaki; Casadesús, Martí y Marimon, Frederic (2004). Incidencia de la certificación ISO 9000 en el rendimiento económico empresarial. Cuadernos de Economía y Dirección de la Empresa, Vol. 18, pp.173-190.

Hernández, Juan.; Stahnke, Wolfgang; Nuñez, Sebastián (2004). Sistemas de gestión de la calidad en el sector agroalimentario. Agroalim [online]. Vol. 9, No.18, pp. 87-93. Disponible en: http://www.scielo.org.ve/ scielo.php? script=sci_arttext\&pid= S1316-35420 $04000100007 \&$ Ing =es\&nrm=iso.

Huarng, Fenghueih; Horng, Ching y Chen, Clave (1999). A study of ISO 9000 process, motivation and performance. Total Quality Management, Vol. 10, No. 7, pp. 1009-1025.

Jones, Robert; Arndt, Guenter y Kustin, Richard (1997). ISO 9000 amongst Australian companies: impact of time and reasons for seeking certification on perceptions of benefits received. International Journal of Quality and Reliability Management, Vol. 14, No. 7, pp. 650-660.

Kaynak, Hale (2003). The relationship between total quality management practices and their effects on firm performance. Journal of Operations Management, Vol. 21, No. 4, pp. 405-435.

Kinnear, Thomas C.; Taylor, James R. (1995). Investigación de Mercados. Un enfoque aplicado. Colombia: McGrawHill, 4ª ed., 1993.

Krasachol, Ladawan; Willey, Phillip y Tannock, James. D.T. (1998). The progress of quality in Tahiland. The TQM Magazine, Vol. 10, No. 1, pp. 40-44.
Lambin, Jacques (1990). Marketing Estratégico. Edit. McGraw Hill Interamericana Editores, S.A.

La fuente, Alberto; Salas, Vicente y Pérez, Ramón (1985). Tipos de empresario y empresa. El caso de las nuevas empresas españolas. Economía Industrial, noviembre-diciembre, pp. 139151. Madrid.

Larsen, Böje y Häversjö, Tord (2001). Management by standards real benefits from fashion. European Journal of Operational Research, Vol. 17, No. 4, pp. 457-480.

Lattin, Gerald (1994). The lodging and food Service industry. The Educational Institute of the American Hotel and Motel Association. Michigan.

Lee, Peter K.C.; To, W.M.; Yu, Billy T.W. (2009). The implementation and performance outcomes of ISO 9000 in service organizations: an empirical taxonomy. International Journal of Quality \& Reliability Management, No. 26, pp. 646-662.

Leung, Hareton K.N.; Chan, Keith C.C. y Lee, T.Y. (1999). Costs and benefits of ISO 9000 series: a practical study. International Journal of Quality and Reliability Management, Vol. 16, No. 7, pp. 675-691.

Magd, Hesham y Curry, Adrienne (2003). ISO 9000 and TQM: are they complementary or contradictory to each other? The TQM Magazine, Vol. 15, No. 4, pp. 244-256.

Mak, Barry L.M. (2011).ISO certification in the tour operator sector. International Journal of Contemporary Hospitality Management, Vol. 23, pp.115-130.

Mallak, Larry A.; Bringelson, Liwana S. y Lyth, David M. (1997). A cultural study of ISO 9000 certification. International Journal of Quality \& Reliability Management, Vol. 14, No. 4, pp. 328-348. 
Marín Vinuesa, Luz María (2009). Enfoques de estudio y modelos de investigación sobre calidad y resultados: una revisión crítica. Revista Cuadernos de Gestión, Vol. 9. No. 2, pp. 89-110.

Marín Vinuesa, Luz María y Gimeno Zuera, Javier (2010). La certificación iso 9000 en el sector industrial del Mueble: evidencias sobre la cultura de calidad Total y las ventajas que la caracterizan. Investigaciones Europeas de Dirección y Economía de la Empresa, Vol. 16, No.1, pp. 77-101.

Marín Vinuesa, Luz María; Gimeno Zuera, Javier y Ruiz-Olalla Corchera, María del Carmen (2007). Influencia de los indicadores no financieros sobre los resultados de las empresas inmersas en procesos TQM: una evidencia empírica. Servicio de Publicaciones, Universidad de La Rioja, España.

Martínez, Manuel (2006). Análisis de la gestión de la calidad total en las entidades gallegas. Un estudio basado en el modelo de Excelencia de la European for Quality Management (EFQM). Tesis Doctoral, Universidad de A Coruña.

McAdam Rodney y Fulton Frances (2002). The impact of the ISO 9000:2000 quality standards in small software firms. Managing Service Quality, Vol. 12 , No. 5, pp. 336-345.

MOKHTAR, Mohd Zulkifli y MUDA, Mohd Shaladdin (2012). Comparative study on performance measure and attributes between ISO and non-ISO certification companies. International Journal of Business and Management, Toronto (Canada), Vol. 7, pp. 185-193.

Muñoz Oñate, Fernando (1994). Marketing Turístico. Edit. Centro de Estudios Ramón Areces. Madrid.

Nicolau, Juan L.; Sellers, Ricardo (2010). The quality of quality awards: disminishing information asymmetries in a hotel chain. Journal of Business Research, Vol. 63, pp. 832-839, 2010.

Nunnally, Jum C. (1998). Psychometric Theory. McGraw-Hill, New York.

Powell, Thomas C. (1995). Total quality management as competitive advantage: $\mathrm{A}$ review and empirical study. Strategic Management Journal, Vol. 16, No. 1, pp. 15-37.

Ragothaman, Srinivasan y Korte, Leon (1999). The ISO 9000 international quality registration: an empirical analysis of implications for business firms. International Journal of Applied Quality Management, Vol. 2, No. 1, pp. 59-73.

Rahman, Shams-ur (2001). Total quality Management practices and business outcome: evidence from a small and medium enterprises in Western Australia. Total Quality Management, March, Vol. 12, No. 2, pp. 35-49.

Rayner, Paul y Porter, Leslie (1991). BS $5759 / 1 S O 9000$ - the experience of small and medium sized firms. International Journal of Quality \& Reliability Management, Vol. 8 , No. 6 , pp. 16-28.

Reed, Richard; Lemak, David y Montgomery, Joseph C. (1996). Beyond process: TQM content and firm performance. Academy of Management Review, Vol. 21, No. 1, pp. 173-202.

Reiner, G. (2002). Analysis of critical factors of company success based on the EFQM excellence model. Proceedings of the 7th World Congress for Total Quality Management, Verona (Italy), Vol. 2, pp. 361-366.

Renner, Peggy (1994). Basic Hotel Front Office Procesures. 3rd. Edition. Van Nostrand Reinhold. New York.

Rubio-Andrada, Luís ; Alonso-Almeida, María; Rodríguez-Antón José Miguel (2011). Motivations and impacts in the firm and stakeholders of quality certification: evidence from small- and me- 
dium-sized service enterprises. Total Quality Management \& Business Excellence, Vol. 22, pp. 833-852.

Santomá, Ricard; Batallé, Pere; Rovira, Xari; Costa, Gerard; Vila, Mar (2008). Determinación de los aspectos de gestión que llevan a la cadena hotelera a mejorar la calidad de su servicio. Leadership-Revista de Circulación Internacional, No. 14, pp. 1-14.

Sánchez Pérez, Manuel y Sarabia Sánchez, Francisco J. (1999). Validez y fiabilidad de escalas, en Sarabia, Francisco .J. (coord..), Metodología para la Investigación en Marketing y Administración de Empresas, Ed. Pirámide, Madrid, pp. 363-393.

Sharma, Divesh S. (2005). The association between ISO 9000 certification and financial performance. International Journal of Accounting, Vol. 40, No. 2, pp. 151-172.

Sheehan, Lorn y Presenza, Angelo (2011). The organizational impacts of quality management in tourism firms: An empirical investigation of the Molise Region, Italy. Tourism, Vol.59, No. 4, pp. 427-446.

Sila, Ismail (2007). Examining the effects of contextual factors on TQM and performance through the lens of organizational theories: An empirical study. Journal of Operations Management, Vol. 25, No. 1, pp. 83-109.

Singels, Jeroen; Ruël, Gwenny y Van De Water, Henny (2001). ISO 9000 series certification and performance. International Journal of Quality \& Reliability Management, Vol. 18, No. 1, pp. 62-75.

Stevenson, Thomas H., Barnes, Frank C. (2001). Fourteen years of ISO 9000 : impact, criticisms, costs, and benefits. Business Horizons, No. May-June, pp. 45-51.

Tan, Lee-Peng y Gilbert Lim-Teck Sie (2001). ISO 9000: the answer for Total Quality
Management implementation? The Malaysian case. Total Quality Management, Vol. 12, No. 2, pp. 223-229.

Tarí, José J. (2000). Calidad Total: fuente de ventaja competitiva. Publicaciones Universidad de Alicante, pp. 1-302.

Tarí, José J. y Molina, Francisco J. (2002). Quality Management Results in ISO 9000 Implementation Practices. International Journal of Quality \& Reliability Management, Vol. 12, No.7, pp.10-27.

Tarí, José J.; Pereira, Jorge (2012). Calidad y rentabilidad. Análisis del certificado $Q$ en las cadenas hoteleras. Universia Business Review, $2^{\circ}$ trimestre,pp. 53-67.

Tarí, José J.; Heras-Saizarbitoria, Iñaki; Dick, Gavin (2012). Internal and External Drivers for Quality Certification in the Service Industry: Do They Have Different Impacts on Success? April Vol.17. Consulta en: http://ssrn.com/ abstract=2041039 or http://dx.doi.org/ 10.2139/ssrn.2041039

Terlaak, Ann y King, Andrew A. (2006). The effect of certification with the ISO 9000 Quality Management Standard: A signalling approach. Journal of Economics Behavior \& Organization, Vol. 60 , pp. 579-602.

Tsiotras, George y Gotzamani, Katerina (1996). ISO 9000 as an Entry Key to TQM: The Case of Greek Industry. International Journal of Quality \& Reliability Management, Vol. 13, No. 4, pp. 64-76.

Vallen, Jerome J. y Vallen, Gary K. (1991). Check-in Check-out. Wm.C. Brown Publishers. United States of America. 4rd. Edition.

Van Der Wiele, Ton.; Williams, Roger y Dale, Barrie (2000). TQM: is a fad, fashion, or fit? Quality Management Journal, Vol. 7, No. 2, pp. 65-67.

Viada-Stenger, M. Clara; Balbastre-Benavent, Francisco y Redondo-Cano, Ana M. 
(2010). The implementation of a quality management system based on the $Q$ Tourist quality standard. The case of hotel sector. Service Business, Vol. 4, No. 3-4. Pp. 177-196.

Vloeberghs, Danield y Bellens, Jan (1996). Implementing the ISO 9000 standards in Belgium. Quality Progress, Vol. 29, No. 6, pp. 43-48.

Wayhan, Victor B.; Kirche, Elias T. y Khumawala, Basheer M. (2002). ISO 9000 certification: The financial performance implications. Total Quality Management, No. 13, Vol. 217-231.

Wenmoth, Bryan y Dobbin, Donald (1994). Experience with implementing ISO 9000. Asia Pacific Journal of Quality Management, Vol. 3, No. 3, pp. 9-27.

Weston, Frederick C. (1995). What do managers really think about registration process? Quality Progress, Vol. 28, No. 10 , pp. 67-73.
Wiele, Ton; Williams, Roger; Brown, Alan; Dale, Barrie (2001). The ISO 9000 series as a tool for organizational change is there a case? Business Process Management Journal, Vol. 7, No. 4, pp. 323-331.

Wongrassamee, S.; Gardiner, Paul y Simmons, John (2003). Performance measurement tools: the Balanced Scorecard and the EFQM Excellence Model. Measuring Business ExceIlence, Vol. 7, No. 1, pp. 14-29.

Yahya, Salleh; Goh, Wee-Keat (2001). The implementation of an ISO 9000 quality system. International Journal of Quality \& Reliability Management, Vol. 18 No. 9, pp. 941-66.

Yung, Winco .K.C. (1997). The values of TQM in the revised ISO 9000 quality system. International Journal of Operations \& Production Management, Vol. 17, No. 2, pp. 221-230. 